\title{
The Study on the Digital Optical Fiber Communication Access Network Technology and its Application
}

\author{
Hechuan ${ }^{1, a}$ \\ ${ }^{1}$ ChongQing College of Electronic Engineering, ChongQing, 401331, China \\ ahechuan023@sina.com
}

Keywords: Digital optical fiber communication; Access network technology; Network

\begin{abstract}
Through the optical fiber transmission technology to realize the network access, it is a kind of communication transmission to optical fiber network for transmission medium means of advanced technology; it is the embodiment of the essence of the optical fiber access. It is large capacity, light weight, and has a good anti-jamming performance and confidentiality and other unique advantages. It is because at this stage of the main causes of communication networks are widely used. The optical fiber communication technology has the very big development, new technologies appear constantly, improve the electrical communication ability, and enlarge the application range of optical fiber communication. This paper summarizes the present situation of the optical fiber communication technology, and introduced the principle of optical fiber access technology, prospects the development characteristics of optical fiber communication technology.
\end{abstract}

\section{Introduction}

It is to implement high-speed transmission of information, to meet the needs of the masses. User access is critical part; optical fiber access network allows people to know the information of all aspects. In the optical fiber access, optical fiber can reach a lot of different position. The optical fiber to the home is the ultimate way of fiber-optic broadband access, it provides full access of light, therefore, can make full use of broadband characteristic of optical fiber, needed to provide users with unlimited bandwidth, fully meet the needs of broadband access. Our country has established the trial network in more than 30 cities and commercial trials network, including residential users and enterprise users, Internet cafes and other application types, also include the carriers dominate, leading, enterprise leading station network operators, real estate developer, dominant and a variety of patterns such as government-led, a good momentum of development.

\section{The current situation of the optical fiber communication technology}

The cities make the optical fiber to the home of the technical standard and construction standards, some cities also formulate the corresponding preferential policies, these are for the development of optical fiber to the home in our country have created good conditions. In the applications of optical fiber to the home, mainly adopts two kinds of techniques, including optical active access technology and passive optical fiber access technology. Of active optical fiber access technology is mainly used as the media converter to realize the user draw the directly connected, it can provide users with high bandwidth access. At present, the domestic technology can provide users with the bandwidth of the FE or GE, for large and medium-sized enterprise users, is the ideal way of access.

Passive optical fiber access technology means that it includes a variety of PON technology, such as APON and EPON and GPON. APON the earliest, "863" projects also successfully developed the APON, but because of many reasons, APON basically do not have application in our country. Now with more is the GEPON in EPON, GEPON in our country is still belong to the achievements of "863" plan, and has been widely used, also exported to Japan, the commonwealth of independent states, Europe, southeast Asia and other overseas countries and regions. GPON due to chip developed late, and is relatively not very mature. Cost also is on the high side, so, started late, but has begun to be applied in our country. Due to its high efficiency, provide TDM business more convenient has good QoS guarantee, therefore, is very promising. EPON and GPON each have 
advantages and disadvantages; EPON residents more suited to the needs of users, and GPON are more suitable for enterprise user access.

\section{The basic structure and characteristics of optical fiber access network}

The basic structure of optical fiber access network. Optical fiber access network is mainly through the optical line terminal the OLT and services of various nodes are mutually connected, and make the optical network unit (ONU) to achieve docking with the user, thus efficient access networks can achieve accurate information transmission function. While the device of optical fiber access network, also has focused on the local system maintenance and remote network monitoring function, light transmission through a transparent and open to form a network which has the function of maintenance management, under the corresponding network protocol specification due to manage the unified network management center. A complete optical fiber access network should include the remote optical network unit device and the local side line terminal equipment. Distal end OLT and ONU in whole by each business access to the network node interface to the user network interface efficient related signaling protocol conversion, including the function of the OLT is for optical fiber access network and the channels of local exchange interface to connect, and through the optical transmission mechanism of high efficient optical network unit connection communication with clients, can realize the function switch exchange with user access to the completely isolated disconnected, and the optical line terminal provides its own equipment and user terminal maintenance and monitoring function, can be directly with the unified local switches in exchange, also can be installed in the remote location. ONU function is to put an end to the optical fiber communication processing signals from OLT, for optical fiber access network provides a rich user interface, make its can be access to many kind of user terminal, can make effective photoelectric conversion function at the same time, and that meet the needs of monitoring and maintenance management.

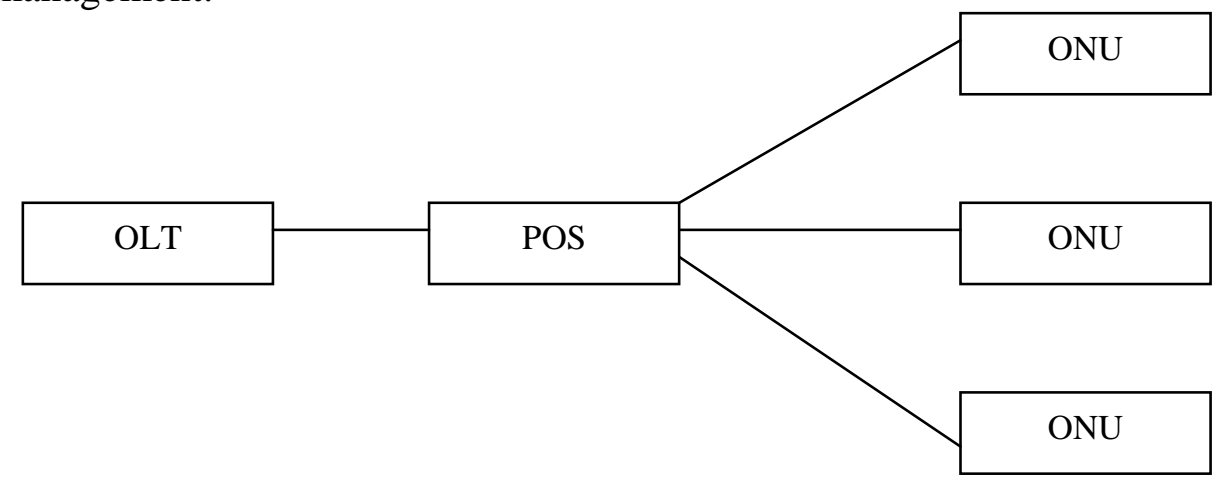

Fig. 1: A typical tree structure

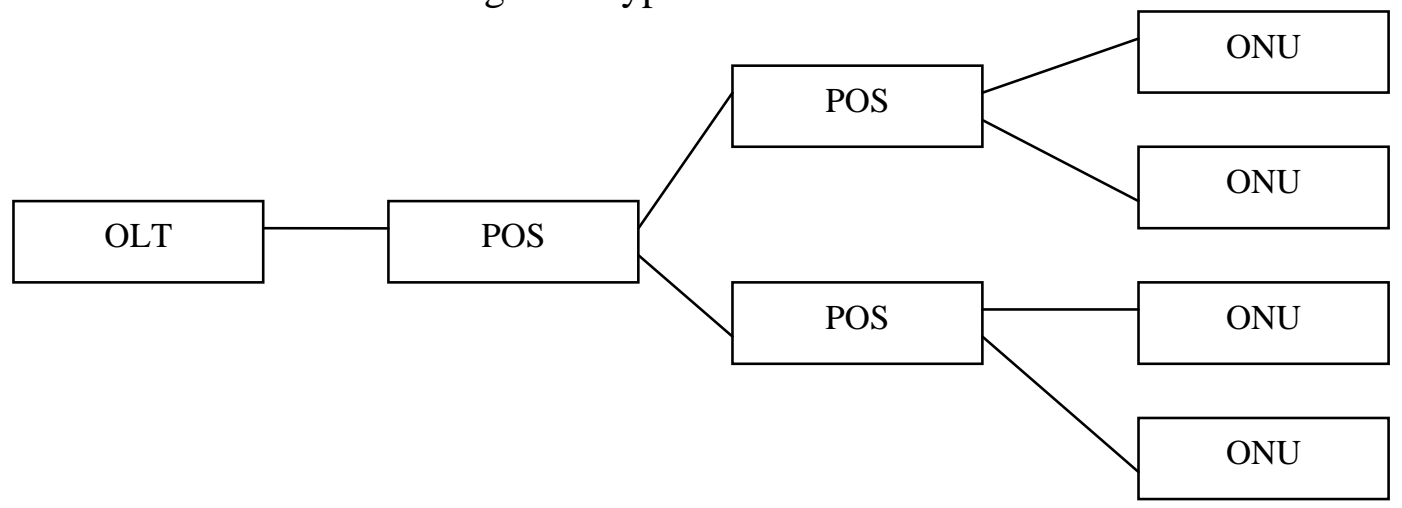

Fig. 2: The multilevel tree

The optical fiber access technology characteristics. Along with the deepening of the urbanization process, the people of all kinds of traffic increases, species also constantly enrich, such as high speed data business, high fidelity music, interactive video multimedia services, etc. To meet these rich network business needs, at present the main application technology including the SDH optical fiber communication transmission, ATM technology and Ethernet technology, according to 
these technologies can effectively build features of active optical fiber access network (AON). If light distribution network by the passive devices to form a whole, without any active category of nodes, then the technology construction of optical fiber access network is a passive optical network (PON). AON network is the lowest cost and easy to implement FTTH access solutions, can easily achieve bidirectional MB broadband access and stability and has a relatively mature technology. But its drawback is that system integration and expansion of construction and development has a certain limitation. Allocated from the system point of view, PON optical networks, the subjects can effectively save fiber resources and network structure level, even in long distance transmission, the system can also provide a two-way high broadband communication ability, therefore access business service variety diverse and operation and maintenance cost is low, is suitable for the regional distribution of users more scattered and users in each region concentrated intensive small areas. Broadband PON technology compared with AON, a high degree of standardization, business good transparency, save the backbone fiber and OLT optical interface, etc. The shortage of the broadband PON technology is the existence of a variety of technical standards to make people difficult to choose, what will be the future development of the mainstream standard have yet to be clearly identified. Moreover, the system requirement with high power laser light emitting module and covers the sudden transceiver ability, and must be integrated with distance measurement, signal complexity functions such as encryption, this will make the system building equipment cost is higher, so the upgrading of its technological development requires us to further in-depth study. Of course there is no denying the fact that the PON technology widely, integrated and optimized development will become the optical fiber access network the inevitable trend of construction.

\section{The development trend of optical fiber communication technology}

To more high speed, large capacity development. A greater capacity for optical fiber communication and the faster speed is people have always been the pursuit of goals. The past the main contradiction of the development history of telecommunications has been can't satisfy people's demand to network and to meet the requirements of the speed of the network. The traditional optical fiber communication is carried out in accordance with the electrical time-division multiplexing way, if transfer can improve the speed of the four times, so every bit costs would fall by $35 \%$ or so, this is also the cause of the optical fiber communication system to increase the transmission speed. High-speed optical fiber system can increase the throughput; this gives the appearance of multimedia condition. The current worldwide installed terminals have been more than 5, 000, in the United States, Europe and Japan and other developed countries have begun to use a lot. Our country also started to experiment, but has been laying optical cable all can not meet the requirements, so want to test first, if the test qualified to open after use. Such as time division multiplexing system also need to increase the rate of transmission, but the time division multiplexing technology to transmit electricity capacity already is the limit of this technique can achieve, so now need to move to reuse technology to solve the shortage of light.

The development of WDM optical fiber communication technology. Due to the potential of time-division multiplexing technology has reached the limit, but the use of broadband resources also amounted to less than $1 \%$, also need to mining. WDM technology is the basis of: if you can put a number of different wavelengths of light signals on an optical fiber to transmit at the same time, this can largely increase the information transmission capacity of optical fiber. Using WDM technology has many advantages, such as broadband huge resources, can be more fully utilized capacity can be expanded several times or even one hundred times; Can save cost; The introduction of broadband method is convenient, can adapt to all kinds of signal rate and modulation mode; Can realize network exchange of transparency.

A lot of benefits because of WDM technology, using the speed is very quickly in the market, rapid development velocity anomaly. The world within the scope of the application of WDM system has been more than 3, 000, the capacity of the practical system can be up to 320 Gbps, lucent, companies in the United States have introduced 80 wavelengths of WDM systems, the total 
capacity can be as high as 400 Gbps. For nearly two years the development of WDM technology is quite rapidly, IP has become the driving force of the business development.

The using optical networking. Has a lot to the transmission capacity of WDM technology, but its reliability and flexibility can't meet the requirements of high. If the light path above can also achieve the function of electric road cross connection and interpolation function, there will be a new power. After the realization of optical networking, has more advantages, can realize the large capacity optical networks, expanding network, increase the number of network nodes and volume, reconstruction of network, the reorganization of the network. Optical networking has so many advantages, the developed countries have begun to invest manpower and funds for scientific research work, in Europe and Japan, have such project is under study. Connected to the Internet will become the new optical transmission technology, highly flexible and large capacity optical networks can lay the foundation for the construction of national information foundation, and development of the national economy and the safety of our country also has the very vital significance.

The use of optical access network. Both from the exchange and transmission, the core of the network are all great changes have taken place. The existing access network of more than $90 \%$ are dominated by twisted-pair copper wire, access network has become the bottleneck which restrict the development of the entire network. There are some technology can solve the problem, such as broadband wireless access systems, but only the means of some transitional, can not fundamentally solve the problem. Access network can reduce the cost of management, reduce the probability of failure, and increase new business, new income increase; Expand the scope of coverage, decrease the node set, the construction of transparent optical networks, and so on. Optical access network, there are 2 classes, respectively for the passive optical network and optical digital loop carrier system. Digital loop carrier system is not a new technology, is in before the technology combined with open interface V5.1 / V5.2, it shows the great vitality. In passive optical network technology of the future, broadband passive optical network will be more and more to exert its advantages, has become the developing direction of the main broadband into technology.

\section{Summary}

Optical fiber communication has become one of the most main information transmission technology, has not yet found the better technology can replace it. Even in the period of the global communications industry in the midst of the downturn, the development of optical fiber communication is never stagnation, in terms of our country, from the point of the development trend of modern communication, optical fiber communication will also become the main trend of future communication.

\section{References}

[1]Assi CM,Yinghua Ye,Sudhir Dixit,Ali MA.Dynamic bandwidth allocation for quality of service over Ethernet PONs. IEEE Journal on Selected Areas in Communications. 2013(12): 232-233.

[2]Gigabit-capable Passive Optical Networks (GPON):Transmission Layer Specification. ITU-TG 984.3. 2014(02): 429-430.

[3]Metro Ethernet Forum.Metro ethernet services definitions phase2.Technical Specification MEF6.1. 2012(10): 1232-1233.

[4]Seong-Ho Jang,Jin-Min Kim,Jong-Wook Jang.A new DBA algorithm supporting priority queues and fairness for EPON. Proceedings of Asia-pacific Optical Communications. 2014(02): 982-983.

[5]Metro Ethernet Forum.Ethernet Services Attributes Phase 2. Technical Specification MEF 10.1. 2011(10): 353-355. 